\title{
Asymptotics of generating the symmetric and alternating groups
}

\author{
John D. Dixon \\ School of Mathematics and Statistics \\ Carleton University, \\ Ottawa, Ontario K2G 0E2 \\ Canada \\ jdixon@math.carleton.ca
}

Submitted: Jul 18, 2005; Accepted: Oct 8, 2005; Published: Nov 7, 2005

MSC 2000: Primary 20B30; Secondary 20P05 05A16 20E05

\begin{abstract}
The probability that a random pair of elements from the alternating group $A_{n}$ generates all of $A_{n}$ is shown to have an asymptotic expansion of the form $1-1 / n-$ $1 / n^{2}-4 / n^{3}-23 / n^{4}-171 / n^{5}-\ldots$. This same asymptotic expansion is valid for the probability that a random pair of elements from the symmetric group $S_{n}$ generates either $A_{n}$ or $S_{n}$. Similar results hold for the case of $r$ generators $(r>2)$.
\end{abstract}

\section{Introduction}

In [5] I proved that the probability that a random pair of elements from the symmetric group $S_{n}$ will generate either $S_{n}$ or $A_{n}$ is at least $1-2 /(\log \log n)^{2}$ for large enough $n$. This estimate was improved by Bovey and Williamson [3] to $1-\exp (-\sqrt{\log n})$. Finally Babai [1] showed that the probability has the asymptotic form $1-1 / n+O\left(1 / n^{2}\right)$. Unlike the earlier estimates, the proof of Babai's result uses the classification of finite simple groups.

Babai's result depends on two elementary results from [5], namely: the probability $t_{n}$ that a pair of elements in $S_{n}$ generates a transitive group is $1-1 / n+O\left(1 / n^{2}\right)$; and the probability that a pair of elements generates a transitive, imprimitive group of $S_{n}$ is $\leq n 2^{-n / 4}$. Using the classification, he shows that the probability that a pair of elements generates a primitive subgroup of $S_{n}$ different from $A_{n}$ or $S_{n}$ is $<n^{\sqrt{n}} / n$ ! for all sufficiently large $n$. Thus the probability that a pair of elements of $S_{n}$ generates a transitive group but does not generate either $S_{n}$ or $A_{n}$ is $O\left(n 2^{-n / 4}+n^{\sqrt{n}} / n !\right)=O\left(n^{-k}\right)$ for all $k$. 
The object of the present paper is to show that there is an asymptotic series of the form $t_{n} \sim 1+\sum c_{k} / n^{k}$ so that

$$
t_{n}=1+c_{1} / n+c_{2} / n^{2}+\ldots+c_{m} / n^{m}+O\left(1 / n^{m+1}\right) \text { for } m=1,2, \ldots .
$$

By what we have just said, the same asymptotic series is valid for the probability that a pair of elements of $S_{n}$ generates either $A_{n}$ or $S_{n}$. We shall also show that this asymptotic series is valid for the probability that a pair of elements in $A_{n}$ generates $A_{n}$.

More precisely, we shall prove the following.

Theorem 1 The probability $t_{n}$ that a random pair of elements from $S_{n}$ generates a transitive group has an asymptotic series of the form described above. The first few terms are

$$
t_{n} \sim 1-\frac{1}{n}-\frac{1}{n^{2}}-\frac{4}{n^{3}}-\frac{23}{n^{4}}-\frac{171}{n^{5}}-\frac{1542}{n^{6}}-\ldots .
$$

The same asymptotic series is valid for the probability that the subgroup generated by a random pair of elements from $S_{n}$ is either $A_{n}$ or $S_{n}$.

Theorem 2 If $a_{n}$ is the number of pairs $(x, y) \in A_{n} \times A_{n}$ which generate a transitive subgroup of $A_{n}$ and $s_{n}$ is the number of pairs $(x, y) \in S_{n} \times S_{n}$ which generate a transitive subgroup of $S_{n}$, then $s_{n}-4 a_{n}=(-1)^{n} 3 \cdot(n-1)$ ! for all $n \geq 1$. Thus for $n \geq 2$ the probability $4 a_{n} /(n !)^{2}$ that a random pair of elements from $A_{n}$ generates a transitive subgroup is equal to $t_{n} \pm 3 /(n \cdot n !)$. Hence the probability that a random pair of elements from $A_{n}$ generates $A_{n}$ has the same asymptotic expansion as given above for $t_{n}$.

Remark 3 The sequence $\left\{t_{n}\right\}$ also appears in other contexts. Peter Cameron has pointed out to me that a theorem of $M$. Hall shows that the number $N(n, 2)$ of subgroups of index $n$ in a free group of rank 2 is equal to $n ! n t_{n}$ (see (1) below and [6]). On the other hand, a result of Comtet [4] (quoted in [8, page 48] and [7, Example 7.4]) implies that $n ! n t_{n}=c_{n+1}$ for all $n \geq 1$ where $c_{n}$ is the number of "indecomposable" permutations in $S_{n}$ (in this context $x \in S_{n}$ is called indecomposable if there is no positive integer $m<n$ such that $x$ maps $\{1,2, \ldots, m\}$ into itself $)$.

We shall discuss a generalisation to more than two generators at the end of this paper.

\section{Lattice of Young subgroups}

In the present section we shall prove Theorem 2. Consider the set $\mathcal{P}$ of all (set) partitions of $\{1,2, \ldots, n\}$. If $\Pi=\left\{\Sigma_{1}, \ldots \Sigma_{k}\right\}$ is a partition with $k$ parts then, as usual, we define the Young subgroup $Y(\Pi)$ as the subgroup of $S_{n}$ consisting of all elements which map each of the parts $\Sigma_{i}$ into itself. The set of Young subgroups of $S_{n}$ is a lattice, and we define an ordering on $\mathcal{P}$ by writing $\Pi \geq \Pi^{\prime}$ when $Y(\Pi) \leq Y\left(\Pi^{\prime}\right)$. Under this ordering the greatest 
element of $\mathcal{P}$ is $\Pi_{1}:=\{\{1\},\{2\}, \ldots,\{n\}\}$ and the least element is $\Pi_{0}:=\{\{1,2, \ldots, n\}\}$. Consider the Möbius function $\mu$ on $\mathcal{P}$ (see, for example, [8, Section 3.7]), and write $\mu(\Pi)$ in place of $\mu\left(\Pi_{0}, \Pi\right)$. By definition, $\mu\left(\Pi_{0}\right)=1$ and $\sum_{\Pi^{\prime} \leq \Pi} \mu\left(\Pi^{\prime}\right)=0$ for all $\Pi>\Pi_{0}$. Example 3.10 .4 of [8] shows that $\mu(\Pi)=(-1)^{k+1}(k-1)$ ! whenever $\Pi$ has $k$ parts.

Now let $f_{A}(\Pi)$ (respectively $f_{S}(\Pi)$ ) be the number of pairs $(x, y)$ of elements from $A_{n}$ (respectively, $S_{n}$ ) such that the parts of $\Pi$ are the orbits of the group $\langle x, y\rangle$ generated by $x$ and $y$. Similarly let $g_{A}(\Pi)$ and $g_{S}(\Pi)$, respectively, be the number of pairs for which the parts of $\Pi$ are invariant under $\langle x, y\rangle$; that is, for which $x, y \in Y(\Pi)$. Every Young subgroup $Y(\Pi)$ except for the trivial group $Y\left(\Pi_{1}\right)$ contains an odd permutation and so we have

$$
g_{A}(\Pi)=\frac{1}{4}|Y(\Pi)|^{2}=\frac{1}{4} g_{S}(\Pi) \text { for } \Pi \neq \Pi_{1} \text { and } g_{A}\left(\Pi_{1}\right)=g_{S}\left(\Pi_{1}\right)=1 .
$$

We also have

$$
g_{A}(\Pi)=\sum_{\Pi^{\prime} \geq \Pi} f_{A}\left(\Pi^{\prime}\right) \text { and } g_{S}(\Pi)=\sum_{\Pi^{\prime} \geq \Pi} f_{S}\left(\Pi^{\prime}\right) .
$$

Since $\mu\left(\Pi_{1}\right)=(-1)^{n+1}(n-1)$ !, the Möbius inversion formula [8, Propositon 3.7.1] now shows that

$$
\begin{aligned}
s_{n} & =f_{S}\left(\Pi_{0}\right)=\sum_{\Pi} \mu(\Pi) g_{S}(\Pi)=4 \sum_{\Pi} \mu(\Pi) g_{A}(\Pi)-3 \mu\left(\Pi_{1}\right) \cdot 1 \\
& =4 f_{A}\left(\Pi_{0}\right)-3 \mu\left(\Pi_{1}\right)=4 a_{n}+(-1)^{n} 3(n-1) !
\end{aligned}
$$

as claimed.

\section{Asymptotic expansion}

It remains to prove Theorem 1 and obtain an asymptotic expansion for $t_{n}=s_{n} /(n !)^{2}$. It is possible that this can be done with a careful analysis of the series $f_{S}\left(\Pi_{0}\right)=\sum_{\Pi} \mu(\Pi) g_{S}(\Pi)$ since the size of the terms decreases rapidly: the largest are those when $\Pi$ has the shapes $[1, n-1],[2, n-2],\left[1^{2}, n-2\right], \ldots$; but the argument seems to require considerable care. We therefore approach the problem from a different direction using a generating function for $t_{n}$ which was derived in [5]. Consider the formal power series

$$
E(X):=\sum_{n=0}^{\infty} n ! X^{n} \text { and } T(X):=\sum_{n=1}^{\infty} n ! t_{n} X^{n} .
$$

Then Section 2 of [5] shows that $E(X)=\exp T(X)$ and so

$$
T(X)=\log E(X) .
$$

We shall apply a theorem of Bender [2, Theorem 2] (quoted in [7, Theorem 7.3]): 
Theorem 4 (E.A. Bender) Consider formal power series $A(X):=\sum_{n=1}^{\infty} a_{n} X^{n}$ and $F(X, Y)$ where $F(X, Y)$ is analytic in some neighbourhood of $(0,0)$. Define $B(X):=$ $F(X, A(X))=\sum_{n=0}^{\infty} b_{n} X^{n}$, say. Let $D(X):=F_{Y}(X, A(X))=\sum_{n=0}^{\infty} d_{n} X^{n}$, say, where $F_{Y}(X, Y)$ is the partial derivative of $F$ with respect to $Y$.

Now, suppose that all $a_{n} \neq 0$ and that for some integer $r \geq 1$ we have: (i) $a_{n-1} / a_{n} \rightarrow 0$ as $n \rightarrow \infty$; and (ii) $\sum_{k=r}^{n-r}\left|a_{k} a_{n-k}\right|=O\left(a_{n-r}\right)$ as $n \rightarrow \infty$. Then

$$
b_{n}=\sum_{k=0}^{r-1} d_{k} a_{n-k}+O\left(a_{n-r}\right) .
$$

Using the identity $(1)$ we take $A(X)=E(X)-1, F(X, Y)=\log (1+Y), D(X)=$ $1 / E(X)$ and $B(X)=T(X)$ in Bender's theorem. Then condition (i) is clearly satisfied and (ii) holds for every integer $r \geq 1$ since for $n>2 r$

$\sum_{k=r}^{n-r} k !(n-k) ! \leq 2 r !(n-r) !+(n-2 r-1)(r+1) !(n-r-1) !<\{2 r !+(r+1) !\}(n-r) !$.

Thus we get

$$
n ! t_{n}=\sum_{k=0}^{r-1} d_{k}(n-k) !+O((n-r) !)
$$

and hence

$$
t_{n}=1+\sum_{k=1}^{r-1} \frac{d_{k}}{[n]_{k}}+O\left(n^{-r}\right)
$$

where $[n]_{k}=n(n-1) \ldots(n-k+1)$. The Stirling numbers $S(m, k)$ of the second kind satisfy the identity

$$
\sum_{m=k}^{\infty} S(m, k) X^{m}=\frac{X^{k}}{(1-X)(1-2 X) \ldots(1-k X)}
$$

where the series converges for $|X|<1$ (see [8, page 34]). Thus for $n \geq k>0$ we have

$$
\frac{1}{[n]_{k}}=\frac{1}{n^{k}(1-1 / n)(1-2 / n) \ldots(1-(k-1) / n)}=\sum_{m=k-1}^{\infty} S(m, k-1) \frac{1}{n^{m+1}} .
$$

This shows that $t_{n}$ has an asymptotic expansion of the form $1+\sum_{k=1}^{\infty} c_{k} n^{-k}$ where $c_{k}=$ $\sum_{i=1}^{k-1} S(k-1, i) d_{i+1}$ since $S(m, 0)=0$ for $m=0$. To compute the numerical values of the coefficients we can use a computer algebra system such as Maple to obtain

$$
D(X)=1 / E(X)=1-X-X^{2}-3 X^{3}-13 X^{4}-71 X^{5}-461 X^{6}-3447 X^{7}-\ldots
$$

and then

$$
\begin{aligned}
t_{n} & \sim 1-\frac{1}{[n]_{1}}-\frac{1}{[n]_{2}}-\frac{3}{[n]_{3}}-\frac{13}{[n]_{4}}-\frac{71}{[n]_{5}}-\frac{461}{[n]_{6}}-\ldots \\
& \sim 1-\frac{1}{n}-\frac{1}{n^{2}}-\frac{4}{n^{3}}-\frac{23}{n^{4}}-\frac{171}{n^{5}}-\frac{1542}{n^{6}}-\ldots
\end{aligned}
$$




\section{Generalization to more than two generators}

In view of the theorem of $\mathrm{M}$. Hall mentioned in Remark 3 there is some interest in extending the analysis for $t_{n}$ to the case of $r$ generators where $r \geq 2$. Let $t_{n}(r)$ be the probability that $r$ elements of $S_{n}$ generate a transitive group (so $t_{n}=t_{n}(2)$ ). A simple argument similar to that in Section 2 of [5] shows that the generating function $T_{r}(X):=\sum_{n=1}^{\infty}(n !)^{r-1} t_{n}(r) X^{n}$ satisfies the equation

$$
T_{r}(X)=\log E_{r}(X)
$$

where $E_{r}(X):=\sum_{n=0}^{\infty}(n !)^{r-1} X^{n}$. Now, following the same path as we did in the previous section, an application of Bender's theorem leads to

$$
t_{n}(r) \sim 1+\sum_{k=1}^{\infty} \frac{d_{k}(r)}{\left([n]_{k}\right)^{r-1}}
$$

where the coefficients $d_{k}(r)$ are given by $1 / E_{r}(X)=\sum_{k=0}^{\infty} d_{k}(r) X^{k}$. For example, we find that

$$
1 / E_{3}(X)=1-X-3 X^{2}-29 X^{3}-499 X^{4}-13101 X^{5}-\ldots
$$

So

$$
\begin{aligned}
t_{n}(3) & \sim 1-\frac{1}{[n]_{1}^{2}}-\frac{3}{[n]_{2}^{2}}-\frac{29}{[n]_{3}^{2}}-\frac{499}{[n]_{4}^{2}}-\frac{13101}{[n]_{5}^{2}} \ldots \\
& \sim 1-\frac{1}{n^{2}}-\frac{3}{n^{4}}-\frac{6}{n^{5}}-\ldots .
\end{aligned}
$$

\section{References}

[1] L. Babai, The probability of generating the symmetric group, J. Combin. Theory (Ser. A) 52 (1989) 148-153.

[2] E.A. Bender, An asymptotic expansion for some coefficients of some formal power series, J. London Math. Soc. 9 (1975) 451-458.

[3] J. Bovey and A. Williamson, The probability of generating the symmetric group, Bull. London Math. Soc. 10 (1978) 91-96.

[4] L. Comtet, "Advanced Combinatorics", Reidel, 1974.

[5] J.D. Dixon, The probability of generating the symmetric group, Math. Z. 110 (1969) 199-205.

[6] M. Hall, Jr., Subgroups of finite index in free groups, Canad. J. Math. 1 (1949) 187190.

[7] A.M. Odlyzko, Asymptotic enumeration methods, in "Handbook of Combinatorics (Vol. II)" (eds.: R.L. Graham, M. Grötschel and L. Lovász), M.I.T. Press and NorthHolland, 1995 (pp. 1063-1229).

[8] R.P. Stanley, "Enumerative Combinatorics (Vol. 1)", Wadsworth \& Brooks/Cole, 1986 (reprinted Cambridge Univ. Press, 1997). 